\title{
Optimization Model for Uncertain Statistics Based on an Analytic Hierarchy Process
}

\author{
Yongchao Hou \\ Department of Mathematical Sciences, Chaohu University, Anhui 238000, China \\ Correspondence should be addressed to Yongchao Hou; yongchaohou1982@163.com
}

Received 6 March 2014; Accepted 20 May 2014; Published 11 June 2014

Academic Editor: Gradimir Milovanović

Copyright (C) 2014 Yongchao Hou. This is an open access article distributed under the Creative Commons Attribution License, which permits unrestricted use, distribution, and reproduction in any medium, provided the original work is properly cited.

\begin{abstract}
Uncertain statistics is a methodology for collecting and interpreting the expert's experimental data by uncertainty theory. In order to estimate uncertainty distributions, an optimization model based on analytic hierarchy process (AHP) and interpolation method is proposed in this paper. In addition, the principle of least squares method is presented to estimate uncertainty distributions with known functional form. Finally, the effectiveness of this method is illustrated by an example.
\end{abstract}

\section{Introduction}

In order to deal with indeterminacy phenomenon in daily life, several axiomatic systems have been constructed. In 1933, Kolmogorov [1] founded probability theory to describe random phenomena by which we can estimate probability distributions when the probability is close enough to real frequency. However, there are some cases where it is difficult to acquire historical data. In these cases, we need domain experts to offer information about the belief degree of each event's occurrence. If we apply probability theory to deal with belief degree, some counterintuitive consequences will follow [2]. In order to manage belief degree reasonably, an axiomatic system named uncertainty theory was proposed by Liu [3] in 2007. In addition, the product uncertain measure was defined by Liu [4] in 2009.

After that, the uncertainty theory and its applications were widely studied by many researchers. Liu [3] presented the concept of uncertain variable and uncertainty distribution to model experts' belief degree. Then, a sufficient and necessary condition of uncertainty distribution was proved by Peng and Iwamura [5] in 2010. In addition, a measure inversion theorem was proposed by Liu [6] from which the uncertain measures of some events can be calculated via the uncertainty distribution. After proposing the concept of independence [4], Liu [6] presented an operational law of uncertain variables. Moreover, in order to describe the average value of an uncertain variable, Liu [6] proposed the concept of expected value and gave a useful formula [7] to calculate the expected values of monotone functions of uncertain variables. Also, Liu [3] presented the concepts of variance, moments, and so forth. A concept of entropy was proposed by Liu [4] to measure the uncertainty of uncertain variables. Dai and Chen [8] verified the positive linearity of entropy and presented some formulas for calculating the entropy of monotone function of uncertain variables. Chen and Dai [9] discussed the maximum entropy principle for selecting the uncertainty distribution that has maximum entropy and satisfies the prescribed constraints. Chen et al. [10] made an extension with a concept of cross-entropy to compare an uncertainty distribution against a reference.

In order to obtain the uncertainty distribution of an uncertain variable when applying uncertainty theory to practice, Liu [6] launched the study of uncertain statistics in 2010. Liu [6] designed a questionnaire to collect the expert's empirical data including lower bound, upper bound, several possible values of an uncertain variable, and their belief degree. Based on these data, the principle of least squares is proposed to calculate the uncertainty distribution with known functional form and unknown parameters. In addition, Wang and Peng [11] suggested the method of moments. Sometimes, the functional form of uncertainty distribution is unknown. In this case, Liu [6] proposed the linear interpolation method. Chen and Ralescu [12] 
suggested spline interpolation method. Moreover, Wang et al. [13] introduced Delphi method to determine uncertainty distributions when multiple domain experts are involved.

In some more complex cases, experts may be not quite familiar with the problem we encountered. This creates incomplete empirical data. As an extension of previous work, this paper proposes an optimization model based on analytic hierarchy process (AHP) and interpolation method to estimate uncertainty distribution with incomplete empirical data. In the first section, some basic concepts about the uncertainty theory and uncertain variables are introduced. In the second section, the AHP method and a monotone piecewise interpolation are presented to construct mathematical programming. In addition, the principle of least squares method is presented to estimate uncertainty distributions with known functional form. In the following section, the effectiveness of this method is illustrated by an example. Finally, conclusions are provided.

\section{Preliminary}

In this section, we will provide a brief introduction of uncertain variable and its expected value, uncertainty distributions, uncertain statistics, and some useful theorems, which will be used throughout this paper.

2.1. Uncertain Variables. A set function $\mathscr{M}$ from a $\sigma$-algebra $\mathscr{L}$ on a nonempty set $\Gamma$ to $[0,1]$ is called an uncertain measure if it satisfies normality axiom, duality axiom, subadditivity axiom (Liu [3]), and product axiom [4]. The triplet $(\Gamma, \mathscr{L}, \mathscr{M})$ is called an uncertainty space. An uncertain variable is defined by Liu [3] as follows.

Definition 1 (see Liu [3]). Let $(\Gamma, \mathscr{L}, \mathscr{M})$ be an uncertainty space. An uncertain variable is a measurable function from an uncertainty space $(\Gamma, \mathscr{L}, \mathscr{M})$ to the set of real numbers; that is, for any Borel set $B$ of real numbers, the set $\xi^{-1}(B)=$ $\{\gamma \in \Gamma \mid \xi(\gamma) \in B\}$ is an event.

For describing uncertain variables, the concept of uncertainty distribution is presented by Liu [3].

Definition 2 (see Liu [3]). The uncertainty distribution $\Phi$ of an uncertain variable $\xi$ is defined by

$$
\Phi(x)=\mathscr{M}\{\xi \leq x\}
$$

for any real number $x$.

Definition 3 (see Liu [3]). An uncertain variable $\xi$ is called normal if its uncertainty distribution is

$$
\Phi(x)=\left(1+\exp \left(\frac{\pi(e-x)}{\sqrt{3} \sigma}\right)\right)^{-1}
$$

denoted by $\mathcal{N}(e, \sigma)$ where $e$ and $\sigma$ are real numbers with $\sigma>0$.
Definition 4 (see Liu [4]). The uncertain variables $\xi_{1}, \xi_{2}, \ldots, \xi_{n}$ are said to be independent if

$$
\mathscr{M}\left\{\bigcap_{i=1}^{n}\left(\xi_{i} \in B_{i}\right)\right\}=\bigwedge_{i=1}^{n} \mathscr{M}\left\{\xi_{i} \in B_{i}\right\}
$$

for any Borel sets $B_{1}, B_{2}, \ldots, B_{n}$ of real numbers.

Theorem 5 (see Liu [3]). Assume that $\xi_{1}, \xi_{2}, \ldots, \xi_{n}$ are independent uncertain variables with regular uncertainty distributions $\Phi_{1}, \Phi_{2}, \ldots, \Phi_{n}$, respectively. If $f\left(x_{1}, x_{2}, \ldots, x_{n}\right)$ is strictly increasing with respect to $x_{1}, x_{2}, \ldots, x_{m}$ and strictly decreasing with respect to $x_{m+1}, x_{m+2}, \ldots, x_{n}$, then $\xi=f\left(\xi_{1}, \xi_{2}, \ldots, \xi_{n}\right)$ is an uncertain variable with inverse uncertainty distribution:

$$
\begin{aligned}
\Psi^{-1}(\alpha)=f( & \Phi_{1}^{-1}(\alpha), \ldots, \Phi_{m}^{-1}(\alpha), \\
& \left.\Phi_{m+1}^{-1}(1-\alpha), \ldots, \Phi_{n}^{-1}(1-\alpha)\right) .
\end{aligned}
$$

To represent the average value of an uncertain variable in the sense of uncertain measure, the expected value is defined as follows.

Definition 6 (see Liu [3]). The expected value of an uncertain variable $\xi$ is defined by

$$
E[\xi]=\int_{0}^{+\infty} \mathscr{M}\{\xi \geq r\} \mathrm{d} r-\int_{-\infty}^{0} \mathscr{M}\{\xi \leq r\} \mathrm{d} r
$$

provided that at least one of the two integrals is finite.

Theorem 7 (see Liu [3]). Suppose that an uncertain variable $\xi$ has an uncertainty distribution $\Phi(x)$. Then,

$$
E[\xi]=\int_{0}^{+\infty}(1-\Phi(x)) \mathrm{d} x-\int_{-\infty}^{0} \Phi(x) \mathrm{d} x .
$$

For calculating the expected value by inverse uncertainty distribution, Liu and $\mathrm{Ha}$ [7] proved the following theorem.

Theorem 8 (see Liu and Ha [7]). Assume that $\xi_{1}, \xi_{2}, \ldots, \xi_{n}$ are independent uncertain variables with regular uncertainty distributions $\Phi_{1}, \Phi_{2}, \ldots, \Phi_{n}$, respectively. If $f\left(x_{1}, x_{2}, \ldots, x_{n}\right)$ is strictly increasing with respect to $x_{1}, x_{2}, \ldots, x_{m}$ and strictly decreasing with respect to $x_{m+1}, x_{m+2}, \ldots, x_{n}$, then the uncertain variable $\xi=f\left(\xi_{1}, \xi_{2}, \ldots, \xi_{n}\right)$ has an expected value

$$
\begin{aligned}
E[\xi]=\int_{0}^{1} f( & \Phi_{1}^{-1}(\alpha), \ldots, \Phi_{m}^{-1}(\alpha), \\
& \left.\Phi_{m+1}^{-1}(1-\alpha), \ldots, \Phi_{n}^{-1}(1-\alpha)\right) \mathrm{d} \alpha .
\end{aligned}
$$

2.2. Uncertain Statistics. Uncertain statistics aims to calculate uncertainty distributions of uncertain variables using the expert's empirical data collected by questionnaires. For example, experts are asked to supply their opinions on an uncertain variable $\xi$ like "how far is it from Beijing to Tianjin?" First, the expert chooses a possible value $x$ (say $110 \mathrm{~km}$ ) that the uncertain variable $\xi$ may take. Second, the expert is asked to give a number to describe the belief degree of $\{\xi \leq x\}$, denoted 
by $\alpha$. Denote this belief degree by $\mathscr{M}\{\xi \leq x\}$ (say 0.6). Then, a pair of the expert's empirical numbers

$$
(x, \alpha)=(110,0.6)
$$

is obtained. Repeating this process, a set of data is obtained as follows:

$$
\left(x_{1}, \alpha_{1}\right),\left(x_{2}, \alpha_{2}\right), \ldots,\left(x_{n}, \alpha_{n}\right),
$$

which meets the following consistence condition:

$$
x_{1}<x_{2}<\cdots<x_{n}, \quad 0 \leq \alpha_{1} \leq \alpha_{2} \leq \cdots \leq \alpha_{n} \leq 1 .
$$

According to the above expert's experimental data, Liu [6] introduced linear interpolation method in which the uncertainty distribution is determined by

$\Phi(x)$

$$
= \begin{cases}0, & \text { if } x<x_{1} \\ \alpha_{i}+\frac{\left(\alpha_{i+1}-\alpha_{i}\right)\left(x-x_{i}\right)}{x_{i+1}-x_{i},}, & \text { if } x_{i} \leq x \leq x_{i+1}, 1 \leq i<n \\ 1, & \text { if } x>x_{n} .\end{cases}
$$

In addition, the principle of least squares is proposed to determine an uncertainty distribution with a known functional form $\Phi(x \mid \theta)$ and an unknown parameter $\theta$ by

$$
\min _{\theta} \sum_{i=1}^{n}\left(\Phi\left(x_{i}, \theta\right)-x_{i}\right)^{2} .
$$

Based on type (9) of expert's experimental data, Wang and Peng [11] presented a method of moments and Wang et al. [13] proposed Delphi method.

\section{AHP Method of Uncertain Statistics}

Based on mathematics and psychology, the analytic hierarchy process (AHP) was developed by Saaty [14] in the 1970s. As a structured method for dealing with complex decisions, the AHP has been widely studied and applied in many fields, such as government management, industry production, business, healthcare, and education. Instead of making a "correct" decision, the AHP helps people seek the one that best suits their perceptions of the problem.

Application of AHP to a decision problem involves four steps.

Step 1. Structure the decision problem in a hierarchy of levels.

Step 2. Make pairwise comparisons by assigning a numerical value.

Step 3. Calculate the maximum eigenvalue, consistency index, and consistency ratio of each comparison matrix and calculate priority weights.

Step 4. Integrate the judgments over various levels of hierarchy to produce an overall priority ranking for alternatives (Figure 1).

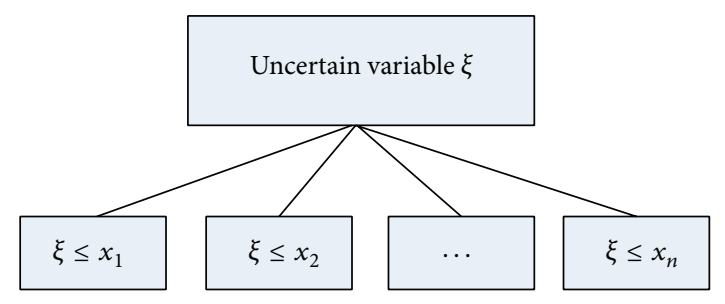

FIGURE 1: AHP model to gather the expert's experimental data.

In order to estimate the distance from Beijing to Tianjin, Chen and Ralescu [12] designed a questionnaire to obtain the expert's opinion. In their questionnaire, the information such as the minimum distance, maximum distance, some other likely distances, and belief degree is needed. In practice, some experts may have no idea about the minimum distance, maximum distance, and the accurate belief degrees. Sometimes, experts only offer some likely distance and the comparative ratios of belief degrees. In this case, the AHP method is taken into account.

Assume that we have obtained a set of expert's experimental data $x_{1}, x_{2}, \ldots, x_{n}$, in which the set $\left\{x_{i} \mid 1 \leq i \leq\right.$ $n\}$ represents the values that the uncertain variable $\xi$ most likely takes or that are appointed by the expert. In order to determine the expert's opinion, a questionnaire survey may be carried out to gather pairwise comparisons of these events $\left\{\xi \leq x_{i}\right\}, i=1,2, \ldots, n$. Let $a_{i j}$ denote the belief degree ratio of two events $\left\{\xi \leq x_{i}\right\}$ and $\left\{\xi \leq x_{j}\right\}$. This means that

$$
a_{i j}=\frac{\mathscr{M}\left\{\xi \leq x_{i}\right\}}{\mathscr{M}\left\{\xi \leq x_{j}\right\}}, \quad(i<j) .
$$

The comparison matrix $A$ is defined as $A=\left(a_{i j}\right)_{n \times n}$, in which $a_{j i}=1 / a_{i j}(i<j)$ and $a_{i i}=1, i=1,2, \ldots, n$. Because of the subjectivity of pairwise comparison, a consistency check is needed. Consistency index (CI) is defined by

$$
\mathrm{CI}=\frac{\lambda_{\max }-n}{n-1},
$$

in which $\lambda_{\max }$ is the maximum eigenvalue of matrix $A$ and $n$ is the number of elements in the comparison.

Each of the numbers in Table 1 is the average of CIs derived from random pairwise judgments. By comparison with $\mathrm{RI}$, the consistency ratio (CR) is defined as

$$
\mathrm{CR}=\frac{\mathrm{CI}}{\mathrm{RI}}
$$

which implies the degree of incredibility.

A CR as high as, say, 90\% would mean that the pairwise judgments are just about random and are completely untrustworthy. By experiments, Saaty and Vargas [15] pointed out that a CR of less than 0.1 is acceptable. If the consistency test fails, pairwise comparisons will be made by experts again.

Assume that $W=\left(w_{1}, w_{2}, \ldots, w_{n}\right)^{\mathrm{T}}$ is the normalized eigenvector belonging to eigenvalue $\lambda_{\max }$. For uncertain statistics, the uncertain measure of uncertain variable $\xi$ less 
TABLE 1: List of random consistency index (RI) values.

\begin{tabular}{ccccccccccc}
\hline$n$ & 1 & 2 & 3 & 4 & 5 & 6 & 7 & 8 & 9 & 10 \\
\hline RI & 0.00 & 0.00 & 0.52 & 0.89 & 1.11 & 1.25 & 1.35 & 1.40 & 1.45 & 1.49 \\
\hline
\end{tabular}

than or equal to $x_{i}$ can be regarded as $w_{i} \kappa, i=1,2, \ldots, n$, where $\kappa$ is an undetermined positive parameter. This means that the expert's experimental data can be represented as

$$
\left(x_{1}, \kappa w_{1}\right),\left(x_{2}, \kappa w_{2}\right), \ldots,\left(x_{n}, \kappa w_{n}\right) .
$$

3.1. Principle of Least Squares. If we know the functional form of an uncertainty distribution $\Phi(x, \theta)$ of uncertain variable $\xi$ with an unknown parameter $\theta$, the principle of least squares will be employed to minimize the error between the expert's experimental data and the uncertainty distribution:

$$
\min _{\theta, \kappa} \sum_{i=1}^{n}\left(\Phi\left(x_{i}, \theta\right)-\kappa w_{i}\right)^{2}
$$

Let $(\widehat{\theta}, \widehat{\kappa})$ be the optimal solution of (17), and then the least squares uncertainty distribution is $\Phi(x, \widehat{\theta}, \widehat{\kappa})$.

3.2. Method of Monotone Spline Interpolation. In order to determine the uncertainty distributions with unknown functional form, an optimization model based on interpolation method is put forward. Since uncertainty distributions are monotone increasing functions, the interpolation should preserve the monotonicity properties. This means that the reconstruct function based on a set of increasing data $\left(x_{1}, \kappa w_{1}\right),\left(x_{2}, \kappa w_{2}\right), \ldots,\left(x_{n}, \kappa w_{n}\right)$ has to be monotone increasing. Monotonicity-preserving interpolation was researched by many scholars. Passow proposed monotone quadratic interpolation [16] and piecewise monotone spline interpolation [17] methods in 1977, respectively. Gasparo and Morandi [18] studied piecewise cubic monotone interpolation with assigned slopes in 1991. Aràndiga et al. [19] presented a new class of nonlinear monotone Hermite interpolation methods in 2013.

The above methods can be used to construct a monotone increasing interpolation function with a parameter $\kappa$ to be determined. Suppose that $S(x, \kappa)$ is a piecewise monotone increasing polynomial (linear, quadratic, or cubic) interpolation:

$$
S(x, \kappa)= \begin{cases}S_{1}(x, \kappa), & x \in\left[x_{1}, x_{2}\right] \\ S_{2}(x, \kappa), & x \in\left[x_{2}, x_{3}\right] \\ \vdots & \vdots \\ S_{n-1}(x, \kappa), & x \in\left[x_{n-1}, x_{n}\right] .\end{cases}
$$

In order to determine the parameter $\kappa$ in (16), we set up the following optimization model:

$$
\begin{aligned}
& \min _{y_{1}, y_{2}, \kappa}\left|\left(x_{1}-y_{1}\right)-\left(y_{2}-x_{n}\right)\right| \\
& \text { subject to } S\left(y_{1}, \kappa\right)=0
\end{aligned}
$$

$$
\begin{aligned}
& S\left(y_{2}, \kappa\right)=1 \\
& \kappa w_{n} \leq 1 \\
& \kappa>0 \\
& y_{1}, y_{2} \in \mathrm{R} .
\end{aligned}
$$

In model (19), $x_{1}$ and $x_{n}$ are the lower and upper numbers that experts offer, while $y_{1}$ and $y_{2}$ represent the lower and upper bounds of the uncertain variable $\xi$, respectively. In the objective function, $\left(x_{1}-y_{1}\right)$ and $\left(y_{2}-x_{n}\right)$ represent the expert's incertitude degree of the lower and upper bounds of $\xi$, respectively. The constant $\widehat{\kappa}$ minimizes the gap between $\left(x_{1}-y_{1}\right)$ and $\left(y_{2}-x_{n}\right)$ for consistency.

Suppose that $\left(\widehat{y_{1}}, \widehat{y_{2}}, \widehat{\kappa}\right)$ is the optimal solution. Then, the uncertainty distribution of $\xi$ is

$$
\Phi(x)= \begin{cases}0, & x \in\left(-\infty, \widehat{y_{1}}\right] \\ S_{1}(x, \widehat{\kappa}), & x \in\left(y_{1}, x_{2}\right] \\ S_{2}(x, \widehat{\kappa}), & x \in\left(x_{2}, x_{3}\right] \\ \vdots ; & \vdots \\ S_{n-1}(x, \widehat{\kappa}), & x \in\left(x_{n-1}, \widehat{y_{2}}\right] \\ 1, & x \in\left(\hat{y}_{2},+\infty\right) .\end{cases}
$$

\section{Example}

Assume that we do not know the real distance between two cities, Beijing and Tianjin, denoted by an uncertain variable $\xi$. Then, a consultation is taken. Suppose that some possible numbers of distance that an expert offered are $120 \mathrm{~km}$, $130 \mathrm{~km}$, and $140 \mathrm{~km}$, denoted by $c_{1}, c_{2}$, and $c_{3}$, respectively. In addition, the pairwise comparison matrix of belief degrees $\{\mathscr{M}\{\xi \leq 120\}, \mathscr{M}\{\xi \leq 130\}, \mathscr{M}\{\xi \leq 120\}\}$ is

$$
A=\left[\begin{array}{lll}
1 & \frac{1}{2} & \frac{1}{3} \\
2 & 1 & \frac{3}{5} \\
3 & \frac{5}{3} & 1
\end{array}\right]
$$

Based on the above expert empirical data (possible numbers and pairwise comparison matrix), we can calculate the uncertainty distribution and expected value of $\xi$ with the following steps.

Step 1 (AHP). The maximum eigenvalue $\lambda_{\max }$ of $A$ is 3.0012 , and the consistency index (CI) is 0.0006 according to formula (14). In 2001, Saaty and Vargas [15] suggested that the random consistency index should be 0.52 when $n=3$ (Table 1). By formula (15), the consistency ratio is 


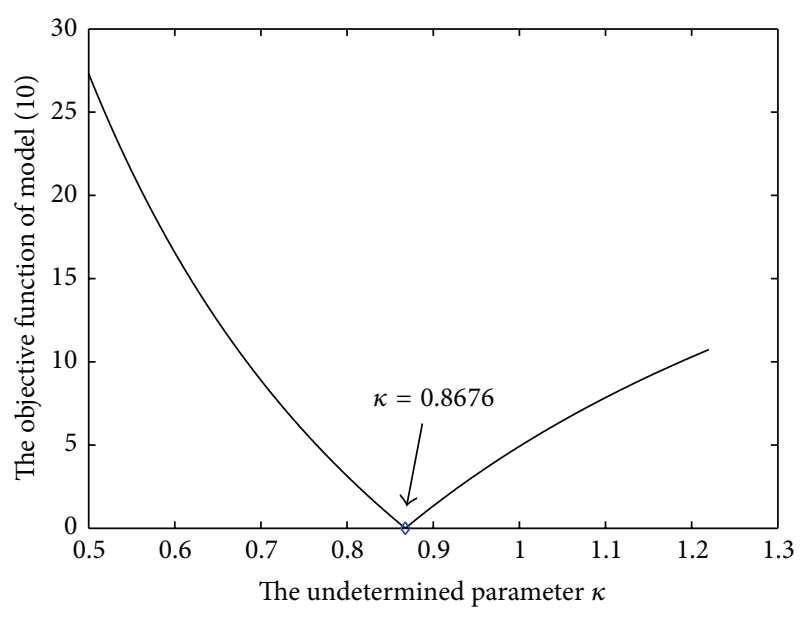

Figure 2: The variation of the objective function with $\kappa$.

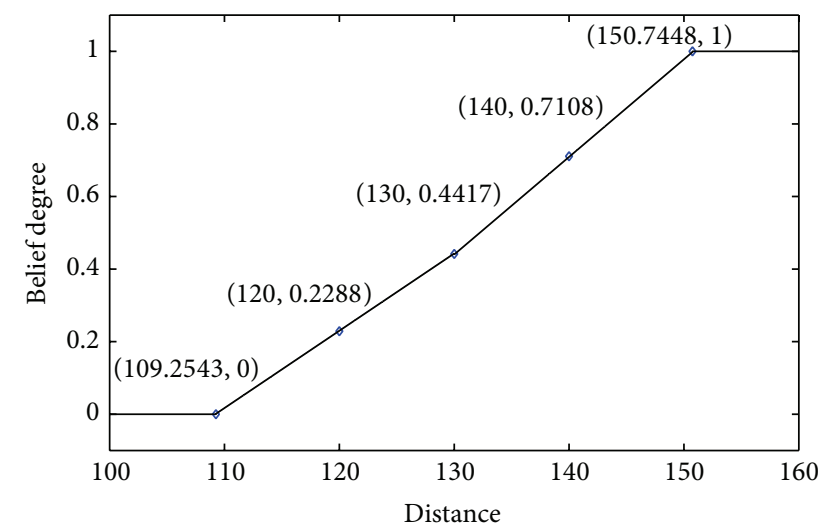

FIgURE 3: The uncertainty distribution of $\xi$.

0.0012 , which is less than 0.10 . The corresponding eigenvector is $(0.2637,0.5091,0.8193)^{\mathrm{T}}$. Assume that the belief degree of $\{\xi \leq 120\},\{\xi \leq 130\}$, and $\{\xi \leq 140\}$ is $(0.2637 \kappa, 0.5091 \kappa, 0.8193 \kappa)^{\mathrm{T}}$, in which $\kappa$ is a positive parameter to be determined.

Step 2 (interpolation). Take piecewise linear interpolation for example. The interpolation function is

$$
S(x, \kappa)= \begin{cases}0.02454 \kappa x-2.6811 \kappa, & x<130 \\ 0.03102 \kappa x-3.5235 \kappa, & x \geq 130 .\end{cases}
$$

Step 3 (optimization). To determine the parameter $\kappa$, we need to solve the following optimization model:

$$
\begin{aligned}
& \min _{y_{1}, y_{2}, \kappa}\left|\left(120-y_{1}\right)-\left(y_{2}-140\right)\right| \\
& \text { subject to } 0.02454 \kappa y_{1}-2.6811 \kappa=0
\end{aligned}
$$

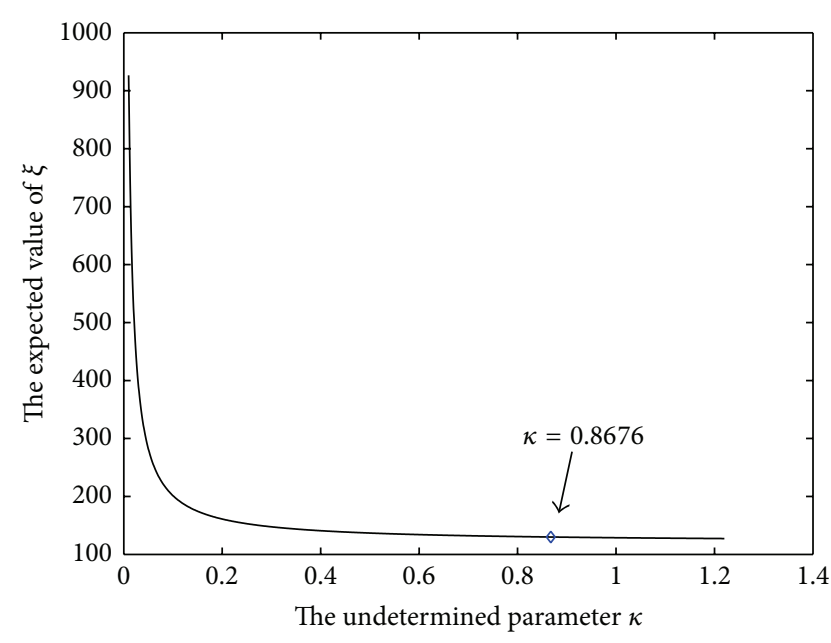

FIGURE 4: The variation of the expected value with $\kappa$.

$$
\begin{aligned}
& 0.03102 \kappa y_{2}-3.5235 \kappa=1 \\
& 0.8193 \kappa \leq 1 \\
& \kappa>0 \\
& y_{1}, y_{2} \in \mathrm{R} .
\end{aligned}
$$

The variation of the objective function with $\kappa$ is shown in Figure 2, and the optimal solution $\left(\widehat{y_{1}}, \widehat{y_{2}}, \widehat{\kappa}\right)^{\mathrm{T}}$ is $(109.2543,150.7448,0.8676)^{\mathrm{T}}$. This means that the minimum distance is $109.2543 \mathrm{~km}$ and the maximum distance is $150.7448 \mathrm{~km}$. In addition, the belief degree of $\xi$ less than or equal to 120,130 , and 140 is $0.2288,0.4417$, and 0.7108 , respectively. The uncertainty distribution (see Figure 3 ) is

$$
\Phi(x)= \begin{cases}0, & x<109.2543 \\ 0.0213 x-2.3261, & 109.2543 \leq x<130 \\ 0.0269 x-3.0570, & 130 \leq x<150.7448 \\ 1, & x \geq 150.7448\end{cases}
$$

The expected value of $\xi$ is $129.9998 \mathrm{~km}$, and its variation with $\kappa$ is shown in Figure 4. From Google Earth, the actual value of the distance from Beijing to Tianjin is $127 \mathrm{~km}$. The relative error is $2.36 \%$.

\section{Conclusions}

In this paper, a new method for estimating uncertainty distributions of uncertain variables was proposed. This method enables experts to supply relative quantities instead of exact values of belief degree. First, we gathered experts' experiential data and checked whether the inconsistency is acceptable. Then, the relative quantity of each belief degree was calculated via AHP. After that, an optimization model was set up to calculate each belief degree based on monotone piecewise interpolation. Finally, an example showed the effectiveness of this method. 


\section{Conflict of Interests}

The author declares that there is no conflict of interests regarding the publication of this paper.

\section{Acknowledgments}

This work was supported by the National Natural Science Foundation of China Grant no. 61273044 and the University Science Research Project of Anhui Province no. KJ2011B105.

\section{References}

[1] A. N. Kolmogorov, Grundbegriffe der wahrscheinlichkeitsrechnung, Julius Springer, Berlin, Germany, 1933.

[2] B. Liu, "Why is there a need for uncertainty theory?" Journal of Uncertain Systems, vol. 6, no. 1, pp. 3-10, 2012.

[3] B. Liu, Uncertainty Theory, Springer, Berlin, Germany, 2nd edition, 2007.

[4] B. Liu, "Some research problems in uncertainty theory," Journal of Uncertain Systems, vol. 3, no. 1, pp. 3-10, 2009.

[5] Z. Peng and K. Iwamura, "A sufficient and necessary condition of uncertainty distribution," Journal of Interdisciplinary Mathematics, vol. 13, no. 3, pp. 277-285, 2010.

[6] B. Liu, Uncertainty Theory: A Branch of Mathematics For Modeling Human Uncertainty, Springer, Berlin, Germany, 2010.

[7] Y. Liu and M. Ha, "Expected value of function of uncertain variables," Journal of Uncertain Systems, vol. 4, no. 3, pp. 181-186, 2010.

[8] W. Dai and X. Chen, "Entropy of function of uncertain variables," Mathematical and Computer Modelling, vol. 55, no. 3-4, pp. 754-760, 2012.

[9] X. Chen and W. Dai, "Maximum entropy principle for uncertain variables," International Journal of Fuzzy Systems, vol. 13, no. 3, pp. 232-236, 2011.

[10] X. Chen, S. Kar, and D. A. Ralescu, "Cross-entropy measure of uncertain variables," Information Sciences, vol. 201, pp. 53-60, 2012.

[11] X. Wang and Z. Peng, "Method of moments for estimating uncertainty distributions," Journal of Uncertainty Analysis and Applications, vol. 2, Article 5, 2014.

[12] X. Chen and D. A. Ralescu, "B-spline method of uncertain statistics with applications to estimate travel distance," Journal of Uncertain Systems, vol. 6, no. 4, pp. 256-262, 2012.

[13] X. Wang, Z. Gao, and H. Guo, "Delphi method for estimating uncertainty distributions," Information, vol. 15, no. 2, pp. 449459, 2012.

[14] T. L. Saaty, The Analytic Hierarchy Process: Planning, Priority Setting, Resource Allocation, McGraw-Hill, New York, NY, USA, 1980.

[15] T. Saaty and L. Vargas, Models, Methods, Concepts \& Applications of the Analytichierarchy Process, Kluwer Academic Publishers, Norwell, Mass, USA, 2001.

[16] E. Passow, "Monotone quadratic spline interpolation," Journal of Approximation Theory, vol. 19, no. 2, pp. 143-147, 1977.

[17] C. de Boor, "Piecewise monotone interpolation," Journal of Approximation Theory, vol. 21, no. 4, pp. 411-416, 1977.

[18] M. G. Gasparo and R. Morandi, "Piecewise cubic monotone interpolation with assigned slopes," Computing, vol. 46, no. 4 , pp. 355-365, 1991.
[19] F. Aràndiga, A. Baeza, and D. F. Yáñez, "A new class of nonlinear monotone Hermite interpolants," Advances in Computational Mathematics, vol. 39, no. 2, pp. 289-309, 2013. 


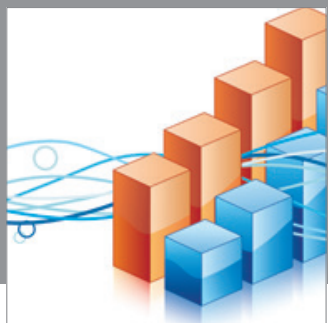

Advances in

Operations Research

mansans

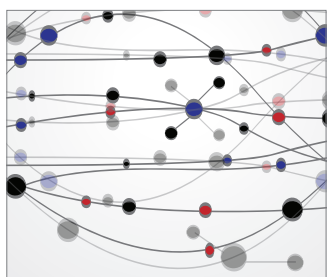

The Scientific World Journal
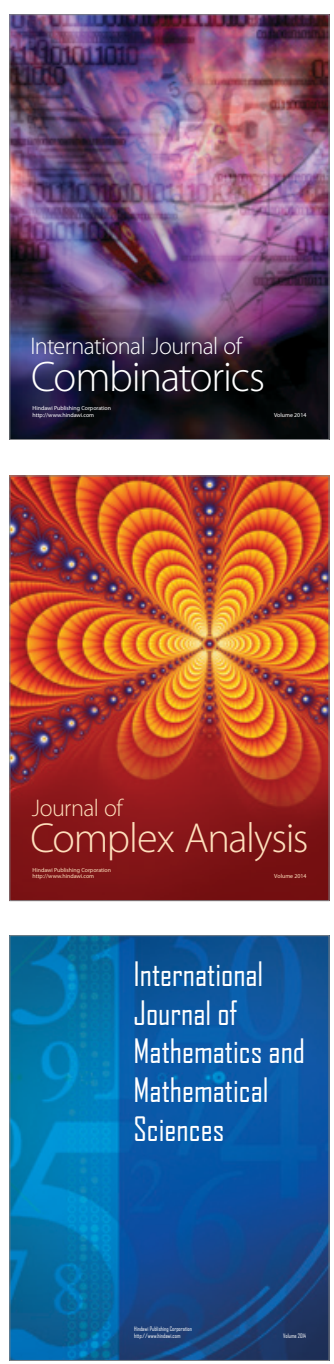
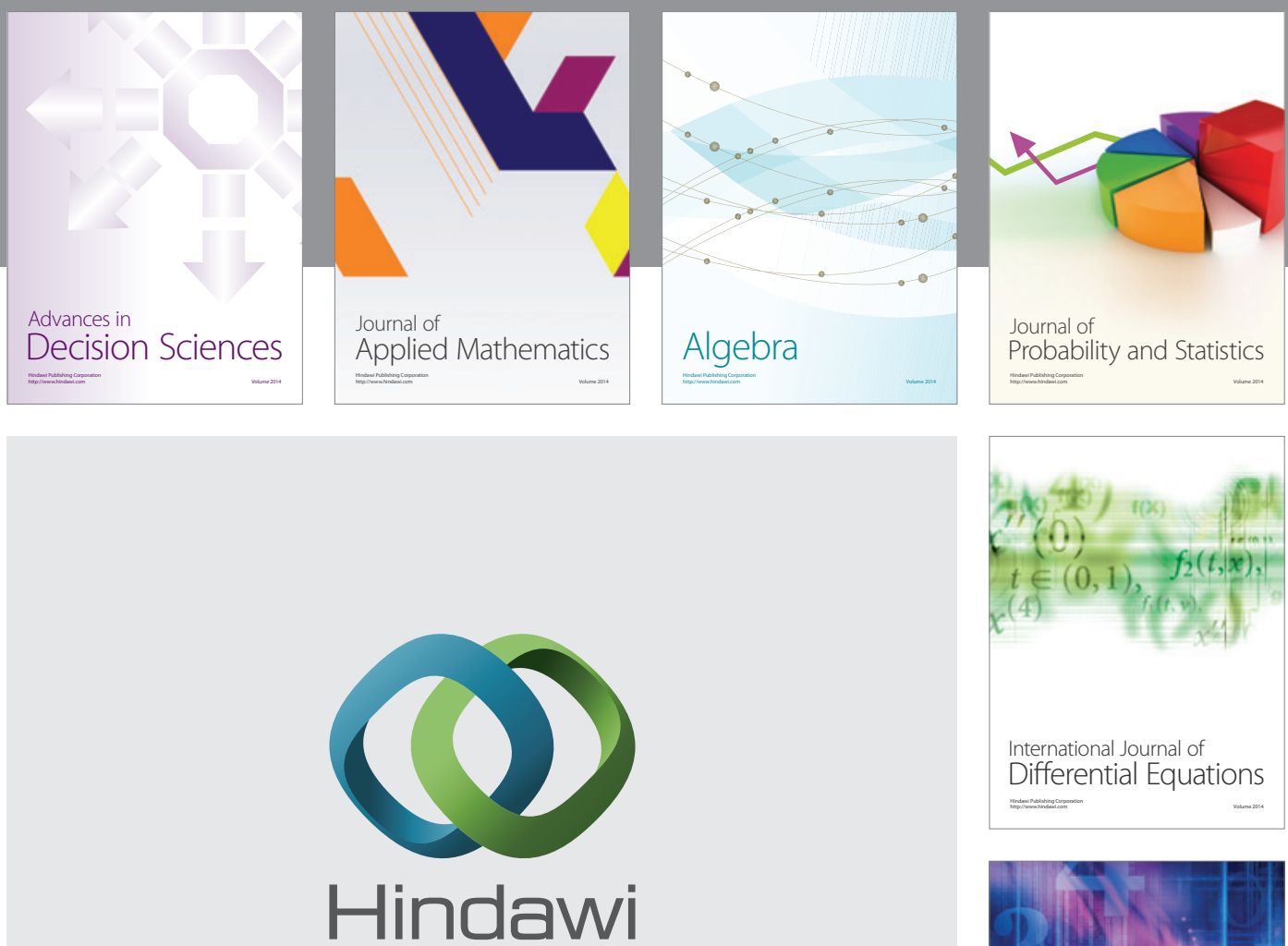

Submit your manuscripts at http://www.hindawi.com
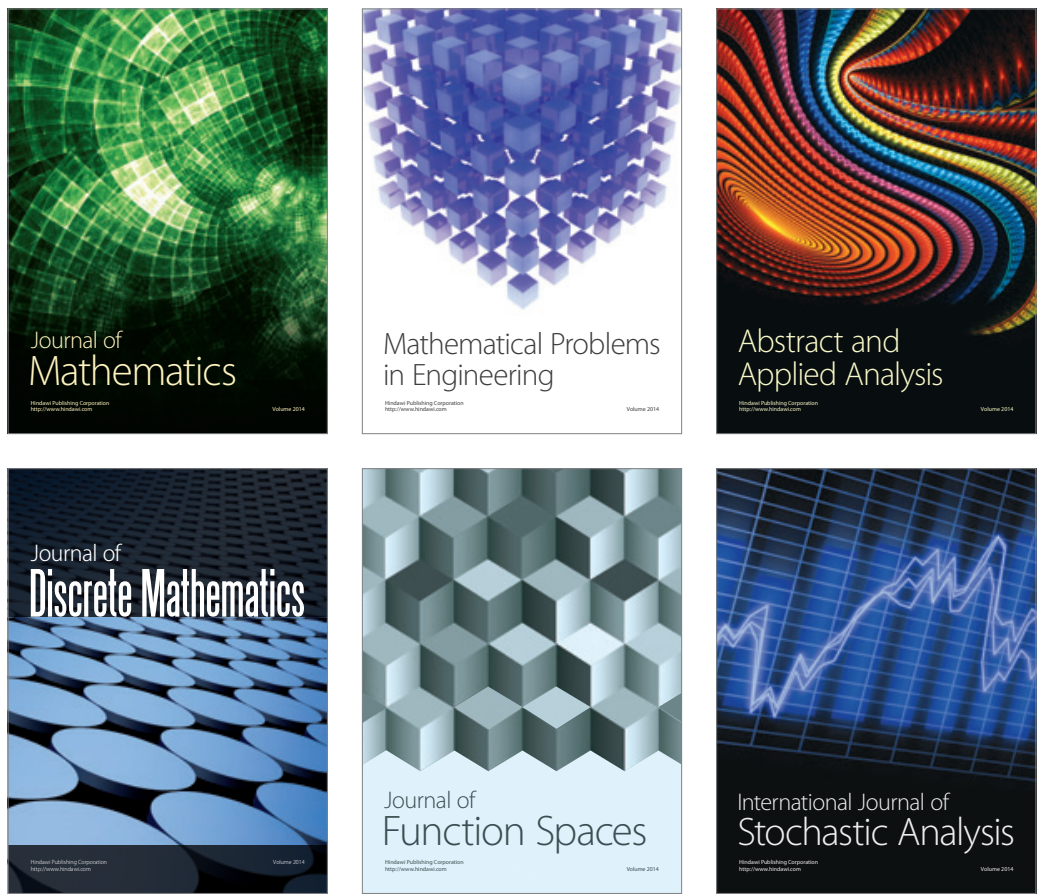

Journal of

Function Spaces

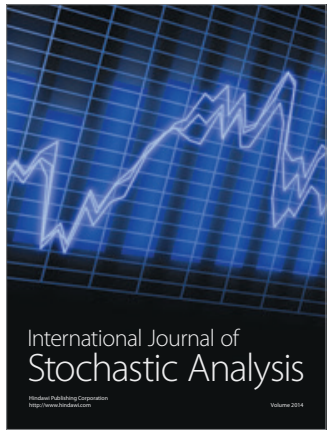

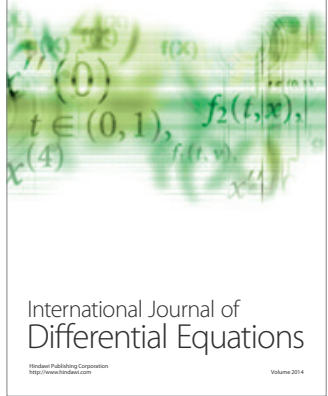
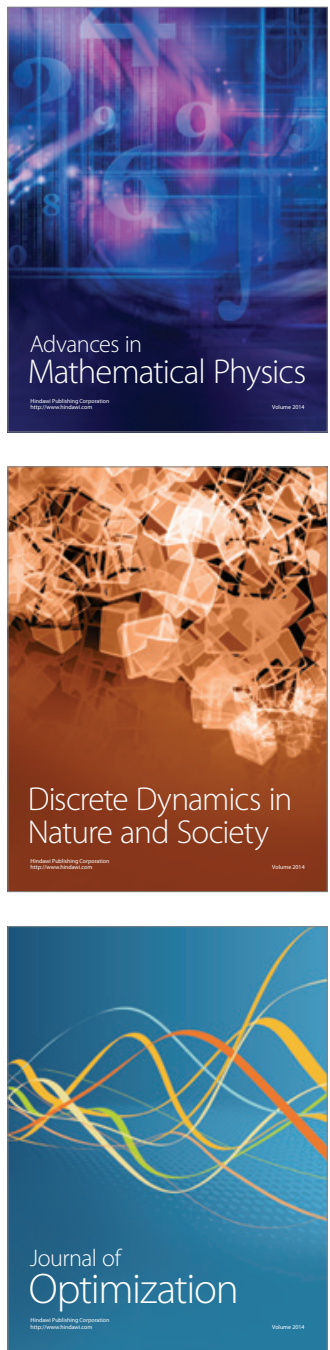